Journal of

Applied

Crystallography

ISSN 0021-8898

Editor: Anke R. Kaysser-Pyzalla

\title{
Characterization of the pore structure of metakaolin-derived geopolymers by neutron scattering and electron microscopy
}

\author{
C. F. Maitland, C. E. Buckley, B. H. O'Connor, P. D. Butler and R. D. Hart
}

J. Appl. Cryst. (2011). 44, 697-707

Copyright (C) International Union of Crystallography

Author(s) of this paper may load this reprint on their own web site or institutional repository provided that this cover page is retained. Republication of this article or its storage in electronic databases other than as specified above is not permitted without prior permission in writing from the IUCr.

For further information see http://journals.iucr.org/services/authorrights.html

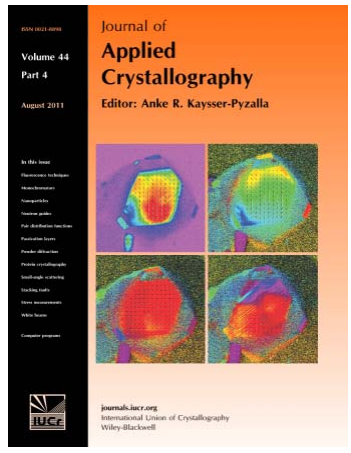

Journal of Applied Crystallography covers a wide range of crystallographic topics from the viewpoints of both techniques and theory. The journal presents papers on the application of crystallographic techniques and on the related apparatus and computer software. For many years, the Journal of Applied Crystallography has been the main vehicle for the publication of small-angle scattering papers and powder diffraction techniques. The journal is the primary place where crystallographic computer program information is published.

Crystallography Journals Online is available from journals.iucr.org 
Journal of

Applied

Crystallography

ISSN 0021-8898

Received 21 March 2010

Accepted 1 June 2011

(C) 2011 International Union of Crystallography

Printed in Singapore - all rights reserved

\section{Characterization of the pore structure of metakaolin-derived geopolymers by neutron scattering and electron microscopy}

\author{
C. F. Maitland, ${ }^{\text {a C. E. Buckley, }}{ }^{\mathrm{a} *}$ B. H. $\mathrm{O}^{\prime}$ Connor, ${ }^{\mathrm{a}}$ P. D. Butler ${ }^{\mathrm{b}}$ and R. D. Hart ${ }^{\mathrm{a}}$ \\ ${ }^{a}$ Centre for Materials Research, Department of Imaging and Applied Physics, Curtin University of \\ Technology, Australia, and ${ }^{\mathbf{b}}$ NIST Centre for Neutron Research, Gaithersburg, USA. Correspon- \\ dence e-mail: c.buckley@curtin.edu.au
}

\begin{abstract}
The pore-solid structure of selected high-compressive-strength metakaolin geopolymers has been characterized to facilitate quantitative prediction of their physical properties. Geopolymers are multiphase materials with pore widths ranging from subnanometre to several tenths of a millimetre. Ultramicrotoming of resin-embedded grains was found to be an effective method for producing electron-transparent sections. Scanning and transmission electron microscopy showed the existence of a bi-level pore system and heterogeneity of the pore morphology. Ultra-small-angle neutron scattering, of sufficiently thin specimens, was found to be useful in detecting the length scales on which statistically significant structural changes occur as the geopolymer chemical composition is varied. Contrast variation experiments confirmed that the small-angle neutron scattering from an Si:Al:Na = 2.5:1:1.2 geopolymer before and after dehydration was dominated by scattering from pores. These experiments suggested the presence of closed (under current experimental conditions) pores in the dehydrated geopolymer. A three-phase analysis was developed for this system, and the scattering of the solid, open pore and closed pore phases was determined as a function of scattering length density $\rho$. The scattering from all three phases had the same $q$ dependence over the range of likely $\rho$ within the uncertainties. A lower limit of $4.21(6) \times 10^{10} \mathrm{~cm}^{-2}$ was determined for the scattering length density $\rho_{\mathrm{w}}$ of the nondehydrated geopolymer by assuming the pore fluid to be water. This scattering length density is significantly higher than the expected value of approximately $3.4 \times 10^{10} \mathrm{~cm}^{-2}$. Small-angle neutron scattering from the dehydrated and nondehydrated $\mathrm{Si}: \mathrm{Al}: \mathrm{Na}=2.5: 1: 1.2$ geopolymer showed that dehydration does not cause a severe change in morphology of the nanoporosity on the length scale probed.
\end{abstract}

\section{Introduction}

The term 'geopolymer' is a name given to a broad class of materials. It was coined by J. Davidovits in the late 1970s to describe a class of materials that he was developing as fire resistant alternatives to conventional organic polymers (Davidovits, 1991). A typical synthesis involved mixing metakaolin (MK, calcined kaolinite) with a highly alkaline potassium or sodium silicate solution, followed by curing at a temperature below $373 \mathrm{~K}$ for several hours. The materials were shown by X-ray diffraction (XRD) to be semi-crystalline to amorphous. Subsequently, similar synthesis conditions have been used with other reactive aluminosilicates [e.g. fly ash (Palomo et al., 1999) and synthetic melt quenched aluminosilicates (Hos et al., 2002)] to make products also referred to as geopolymers. Alternative names used in the literature for describing geopolymers include 'low-temperature aluminosilicate glass', 'alkali-activated cement', 'geocement', 'hydroceramic', 'alkali-bonded ceramic', 'aluminosilicate inorganic polymer' (AIP) and 'low-temperature inorganic polymer glass' (LTIPG or IPG) (Rahier et al., 1995; Rowles, 2004; Duxson, Fernandez-Jimenez et al., 2007).

Geopolymers can be synthesized to have physical properties that include high compressive strength (Rowles \& O'Connor, 2003); little or no shrinkage during curing; and resistance to acidic environments (Palomo et al., 1999), phosphates (Palomo et al., 1999) and heat (Davidovits, 1991). The compressive strength is strongly dependent on processing condition changes and is the most commonly reported physical property. Potentially, they are suitable for many applications such as an alternative to ordinary Portland cement (OPC), high-temperature composites, radioactive waste encapsulation, castable ceramics and others (Davidovits, 1991; Duxson, Fernandez-Jimenez et al., 2007). Fly-ashbased geopolymer concrete as an OPC concrete alternative has been manufactured on a commercial scale (Hardjito et al., 2005; Gourley \& Johnson, 2005; Lukey \& Van Deventer, 2005) 
and is attractive environmentally from a $\mathrm{CO}_{2}$ emissions perspective. Further scientific understanding of geopolymer synthesis, structure and durability is required for ongoing assessment of its suitability for widespread implementation.

A convenient way to conceptualize metakaolin-based geopolymers is to consider them as a composite composed of partially reacted metakaolin, solid reaction product and pore space. Some of the pore space is filled with an alkaline aqueous solution. The solid reaction product is usually amorphous and is the only true inorganic polymer component. The open-pore volume fraction (the volume fraction of the sample filled by a fluid in which the sample is immersed) has been reported between $<1$ and 40\% (Subaer, 2004; Perera et al., 2003, 2007; Duxson et al., 2005; Kriven, 2004) and is dependent on starting-material composition and processing conditions. Pores with 'sizes' ranging from subnanometre (Vance et al., 2007) to 0.5 millimetres have been reported, the largest voids being attributed to air trapped during processing. A significant volume of micro- and mesopores are observed in most samples (Duxson et al., 2005; Kriven et al., 2004; Bell \& Kriven, 2004). For example, the Barret-Joyner-Halenda (BJH) pore-volume distribution reported by Duxson et al. (2005), of an Na-based metakaolin geopolymer with $\mathrm{Si}: \mathrm{Al}=$ 2.1, suggests that more than half of the pore space has a diameter of less than $10 \mathrm{~nm}$. Using porosimetry, Kriven and co-workers (Kriven, 2004) measured the average pore diameter of a K-based metakaolin geopolymer to be $7 \mathrm{~nm}$. Transmission electron micrographs have indicated the existence of a pore system arising from aggregates of geopolymer particulates of around $5 \mathrm{~nm}$ in diameter (Kriven et al., 2004; Bell \& Kriven, 2004; Duxson et al., 2005).

In this article the pore structure of high-strength sodiumbased metakaolin geopolymers previously reported by Rowles and co-workers (Rowles \& O'Connor, 2004, 2009; Rowles et $a l ., 2007,2003)$ is examined. A prime aim of those studies was to determine the influence of $\mathrm{Si}, \mathrm{Al}$ and $\mathrm{Na}$ composition on the compressive strength over a large range of Si:Al:Na molar ratios. The highest compressive strength measured was 64 (3) $\mathrm{MPa}$, for a ratio of $\mathrm{Si}: \mathrm{Al}: \mathrm{Na}=2.5: 1: 1.26$. The structure was examined to explain changes in compressive strength, with an emphasis on the concentration of partially reacted MK and chemical composition on the micrometre scale, and the atomistic structure as measured by X-ray scattering and NMR. Duxson and co-workers (Duxson, Mallicoat et al., 2007; Duxson et al., 2005) continued this work on similar metakaolin geopolymers by considering changes in Young's modulus as well as compressive strength. Their focus was on pore structure changes observable in SEM micrographs of polished sections and using nitrogen adsorption. Rowles \& O'Connor and Duxson et al. proposed reasons for changes in compressive strength in terms of the structural characteristics. Quantitative predictions of the compressive strength or Young's modulus were not made from structural information. Empirical equations fitted to compressive strength data by Subaer (2004) showed that compressive strength decreases with an increase in open pore volume fraction. The complex multiscale structure of geopolymers makes the accurate calculation of these physical properties from structural information very difficult.

An aim of the current studies is to eventually construct three-dimensional structural models of the geopolymers from which physical properties can be calculated. These models will also allow a precise morphological description of the multiscale geopolymer structure via simulation of electron microgaphs and of ultra-small-angle and small-angle neutron scattering (USANS and SANS) curves. Model construction and validation requires electron micrographs of cross sections of the material, as well as knowledge of the following: the general structural characteristics such as indentification and concentration of the different phases present, and the size range and heterogeneity of pore systems; the scattering length density of the solid geopolymer phase; the influence of dehydration on the structure; the accessibility of the pore systems to water; and the dominant source of USANS and SANS. This article reports the determination of this structural information.

\section{Experimental}

\subsection{Sample production}

The geopolymers were produced using the same materials and methods as described by Rowles \& O'Connor (2003), which are summarized here. Metakaolin was produced by heating kaolinite (Kingwhite 65, Unimin Australia Ltd) at $1023 \mathrm{~K}$ for $24 \mathrm{~h}$. This was mixed by hand with a sodium silicate solution (NaOH, Sigma Chemicals Pty Ltd, Australia; Silica fume, Australian Fused Materials Pty Ltd, Australia) and poured into cylindrical polycarbonate moulds. These moulds were then sealed and placed in an oven at $348 \mathrm{~K}$ for $24 \mathrm{~h}$ for the paste to cure.

Four geopolymer samples were examined: 2.5/1.26, 2.5/1.2, 2.0/1.0 and 2.8/1.4. Samples are referred to by a nominal ratio in this report, e.g. sample 2.8/1.4 refers to the geopolymer with molar ratios (calculated from masses of starting materials) of $\mathrm{Si}: \mathrm{Al}=2.77(2), \mathrm{Na}: \mathrm{Al}=1.35$ (2). This is similar to the naming convention used by Rowles (2004), who also gives the compressive strengths of similarly prepared geopolymers. The production method of 2.5/1.26 and 2.5/1.2 was identical except that 2.5/1.26 was not cast on a vibrating table.

\subsection{Scanning electron microscopy (SEM)}

Fracture surfaces were produced by snapping thin pieces of geopolymer. Within 30 min of being generated, these surfaces were coated with a thin layer of gold using a sputter-type coater. SEM micrographs were recorded on a Phillips XL30 microscope fitted with a tungsten wire filament. The micrograph displayed in Fig. 1(a) was recorded using an accelerating voltage of $20 \mathrm{kV}$ and a working distance of $10.1 \mathrm{~mm}$.

\subsection{Transmission electron microscopy (TEM)}

Grains of geopolymer were removed with a stainless steel scalpel blade and those of the smallest visible size placed in a polyethylene embedding capsule. This mould was then filled 
with a 'hard' formulation of low-viscosity Spurr's resin (Spurr, 1969; ProSciTech, AUS). The resin was cured at $343 \mathrm{~K}$ after being left to infiltrate the geopolymer grains for several days. Sections were cut on a Reichert ultramicrotome using a diamond knife (Micro Star Tech, USA) with an included angle of $45^{\circ}$. The colour of these sections when floating on water indicated that their thickness was approximately $100 \mathrm{~nm}$. Sections were mounted on 'holey' carbon film coated copper grids. TEM micrographs were recorded using a Jeol-2011

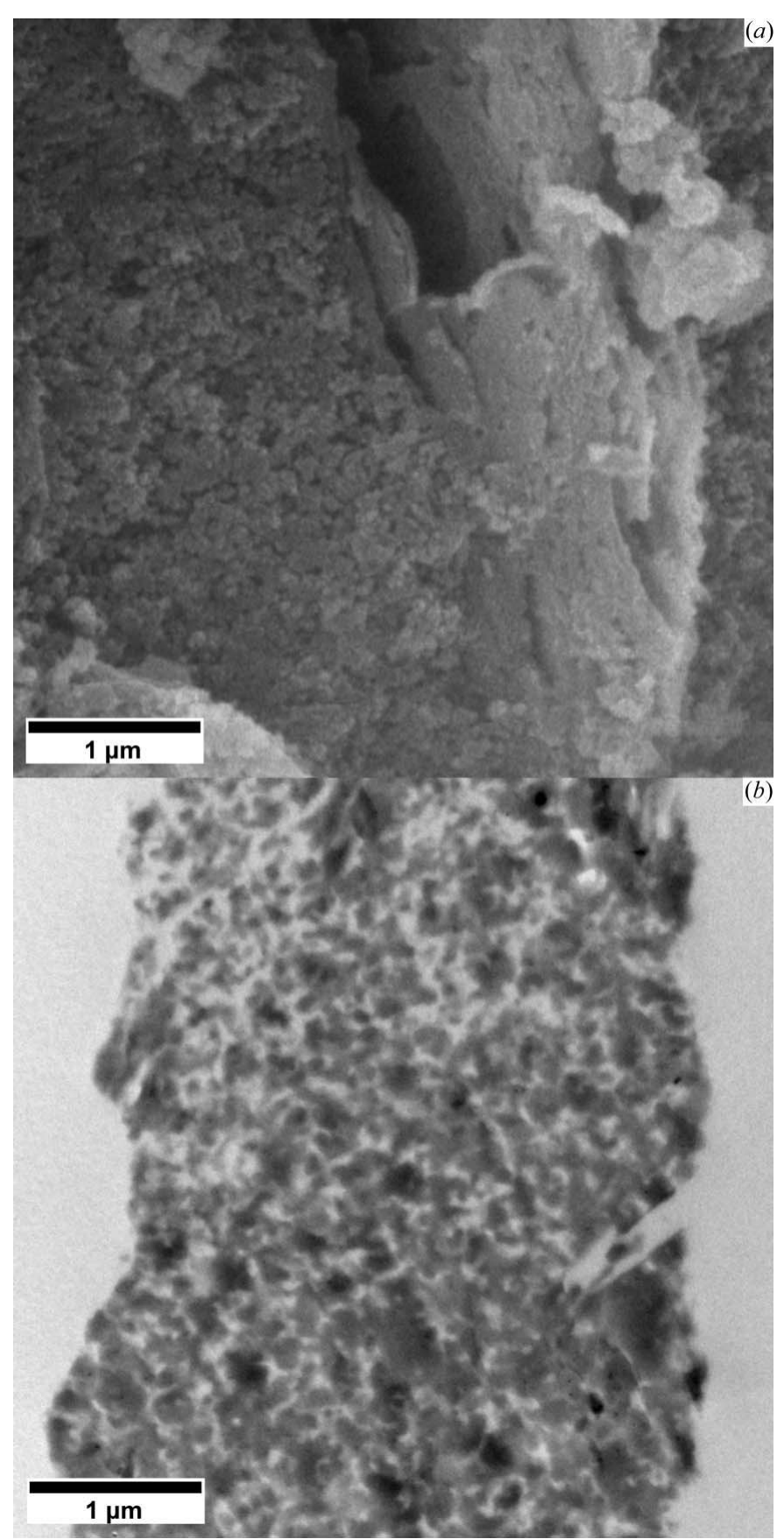

Figure 1

SEM (a) and TEM (b) micrographs recorded from sample 2.5/1.26, showing the level 1 pore system of the matrix. The plate-shaped feature in (a) is a remnant of a metakaolin particle. microscope fitted with an $\mathrm{LaB}_{6}$ electron source and Gatan CCD camera, operated at an accelerating voltage of $200 \mathrm{kV}$.

\subsection{Small-angle neutron scattering}

Discs were cut from the cylindrical samples with a nickelbonded diamond wafering blade (UKAM, USA), and some were thinned further by mounting on a custom-made tool and polishing with $\mathrm{SiC}$ abrasive paper. Final specimen thicknesses ranged between 0.5 and $1 \mathrm{~mm}$. The disc-shaped specimens were sandwiched between $1 \mathrm{~mm}$-thick quartz windows and mounted in sealable cells. For contrast variation measurements, the specimens were placed horizontally in the sample holder and solvent was added dropwise to the centre of the disc.

SANS was recorded from two sets of specimens to allow for a comparison between dehydrated and nondehydrated geopolymer 2.5/1.2. The dehydrated specimens were dried in an air-filled oven at $423 \mathrm{~K}$ for more than $12 \mathrm{~h}$. After removal from the oven, the dehydrated specimens were immediately mounted in air-tight sample cells or left to cool for several minutes then immersed in solvents of $\mathrm{H}_{2} \mathrm{O} / \mathrm{D}_{2} \mathrm{O}$. The nondehydrated specimens were left to dry in the air-conditioned atmosphere (the temperature varied between 288 and $290 \mathrm{~K}$ ) of the SANS instrument guide hall for more than $12 \mathrm{~h}$. Immediately before saturation of the nondehydrated specimens in $\mathrm{H}_{2} \mathrm{O} / \mathrm{D}_{2} \mathrm{O}$ solvents, the rate of mass decrease for all specimens was less than $0.001 \mathrm{~g} \mathrm{~h}^{-1}$. Sample masses ranged between 0.561 and $0.611 \mathrm{~g}$ owing to slight differences in their dimensions.

All specimens were weighed and their thickness measured after (i) initial drying or dehydration, (ii) being saturated with their respective solvents, (iii) redrying (after being saturated and SANS recorded) in the air of the SANS instrument guide hall for more than $12 \mathrm{~h}$, and (iv) final redrying in air at $423 \mathrm{~K}$ for $3 \mathrm{~h}$. Four hours after measurement (iii) the relative humidity (RH) and temperature of the air in the guide hall were $50 \%$ and $289.3 \mathrm{~K}$. Measurement (i) of the nondehydrated specimens occurred $53 \mathrm{~h}$ before the relative humidity and temperature were recorded. Owing to the possibility of variation in relative humidity in the time between the mass and the relative humidity measurements, the absolute hydration state of the nondehydrated specimens was not known. However, it was assumed that the set of nondehydrated specimens used for the contrast variation experiments were in the same hydration state as each specimen of this set had been exposed to the same atmosphere for more than $12 \mathrm{~h}$, and all specimens were saturated with solvent over a period of less than $10 \mathrm{~min}$.

Small-angle neutron scattering patterns were recorded with the AUSANS instrument at ANSTO, Lucas Heights, Australia. Neutrons with a mean wavelength $\lambda=0.35 \mathrm{~nm}$ were used. The available $q$ range with the detector offset was 0.14 $2.0 \mathrm{~nm}^{-1}$. Two-dimensional raw data were corrected for background contributions and detector sensitivity then radially averaged. The resulting one-dimensional data were converted to an absolute scale using a secondary silica stan- 
dard. These absolute data are denoted as $I(q)$, where $q=4 \pi \sin \theta / \lambda, 2 \theta$ being the scattering angle.

\subsection{Ultra-small-angle neutron scattering}

Preliminary USANS measurements suggested the specimens would have to be less than approximately $0.4 \mathrm{~mm}$ thick to prevent severe multiple scattering effects, and hence specimens between 0.33 and $0.35 \mathrm{~mm}$ thick were prepared. After thinning, the specimens were left to dry in an airconditioned room for several days. The temperature and relative humidity $4 \mathrm{~h}$ prior to sealing were $296.7 \mathrm{~K}$ and $42 \%$, and had changed to $296.4 \mathrm{~K}$ and $64 \%$ at the time of sealing. Each disc-shaped USANS specimen was sandwiched between two $1.6 \mathrm{~mm}$-thick quartz windows (Esco Products Inc., USA); the window-specimen-window stack was then mounted inside an aluminium ring and sealed with epoxy.

USANS data were collected with the Bonse-Hart-type double-crystal diffractometer at the NIST Center for Neutron Research (NCNR; Barker et al., 2005). Neutrons with a mean wavelength $\lambda=0.238 \mathrm{~nm}$ were used. The USANS data $I_{\mathrm{s}}(q)$

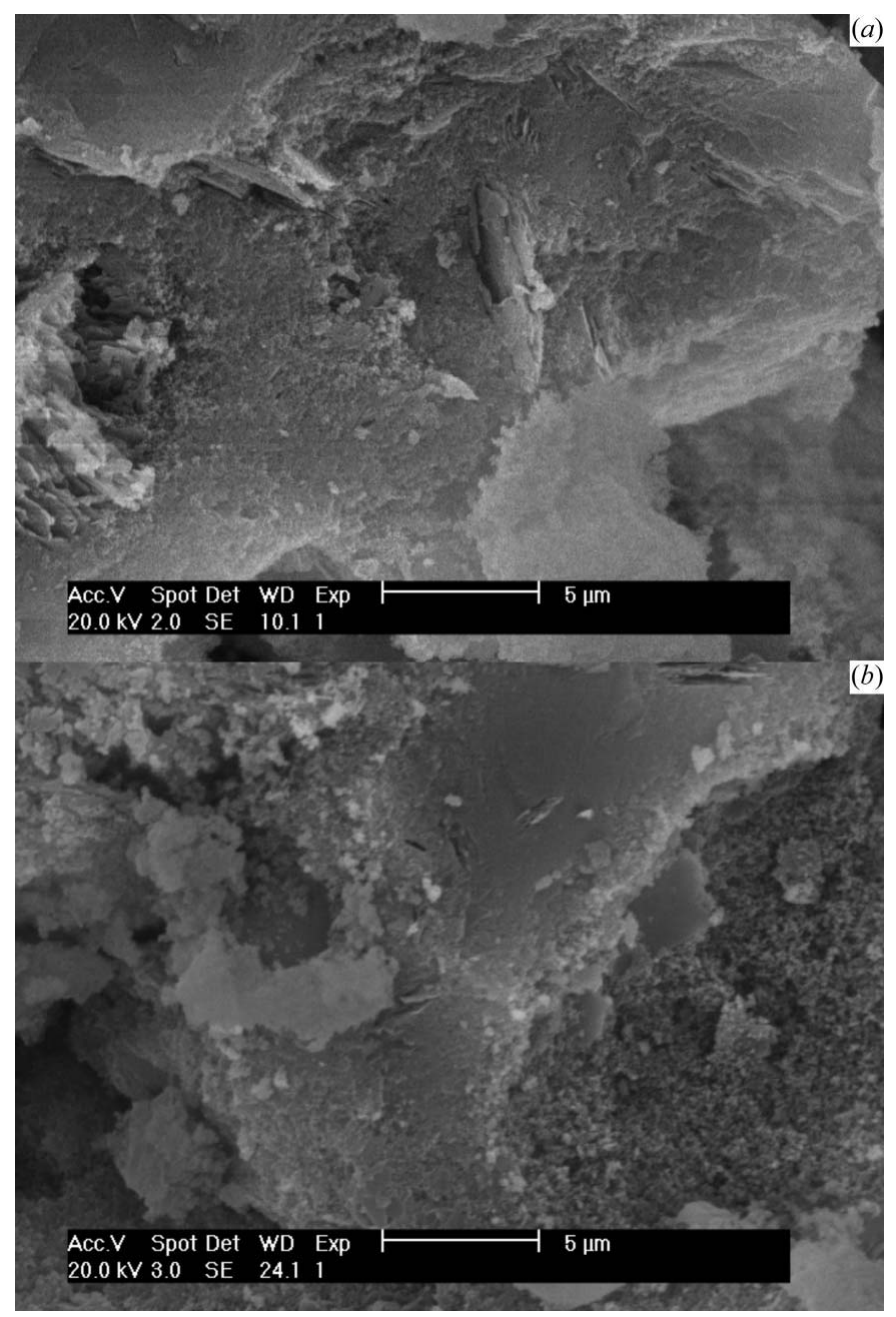

Figure 2

SEM micrographs of different regions of the fracture surface of geopolymer $2.5 / 1.26$, showing the variation in morphology of the level 1 pore system. are 'slit smeared', whereas the SANS data $I(q)$ were 'pin hole smeared'. The two data types can only be directly compared if they have both been desmeared. The slit smearing is described by (Kline, 2006)

$$
I_{\mathrm{s}}(q)=\left(1 / \Delta q_{\mathrm{v}}\right) \int_{0}^{\Delta q_{\mathrm{v}}} I_{0}\left[\left(q^{2}+q_{\mathrm{v}}^{2}\right)^{1 / 2}\right] \mathrm{d} q_{\mathrm{v}} .
$$

where $I_{0}(q)$ is the intensity before any smearing. The USANS data presented in $\$ 3.2$ were collected with the maxiumum extent of $q$ resolution in the vertical direction $\Delta q_{\mathrm{v}}=$ $1.17 \mathrm{~nm}^{-1}$. Software packages developed at the NIST Centre for Neutron Research (Kline, 2006) were employed for reduction of SANS and USANS data. The SANS reduction package had been modified by J. Schulz to read raw AUSANS data.

\section{Results and discussion}

\subsection{SEM and TEM: Surface and cross sectional observation of pore morphology}

Starting at the millimetre level, the largest inhomogeneities in metakaolin geopolymers are usually isolated, globular voids formed by air trapped during casting. The maximum size and concentration of these voids depends upon any action taken to remove them. Kriven et al. (2003) report having most success by curing the geopolymer paste in a high-pressure autoclave. Sample 2.5/1.2, cast on a vibrating table, had voids less than $0.5 \mathrm{~mm}$ in size. At a resolution of several micrometres these geopolymers appear to be remnants of MK particles dispersed amongst a matrix of a reaction product. The reader is referred to Rowles \& O'Connor $(2004,2009)$ for SEM micrographs and elemental analyses of MK geopolymers mounted in epoxy and polished with mats charged with abrasive. These studies found the remnants of MK to be chemically dissimilar to the MK precursor.

As shown in Fig. 1(a), at higher resolutions it becomes apparent that the matrix of sample $2.5 / 1.26$ is porous. The pores visible at this resolution are referred to in this article as level 1 pore structure. The appearance of the level 1 poresolid structure in some regions is vaguely similar to that of agglomerations of spheres coalesced to different degrees. This structure is similar to that in other metakaolin (Bell et al., 2006; Bell \& Kriven, 2004; Kriven et al., 2003) and synthetic (Hos et al., 2002) geopolymers. The appearance and size of this structure varies significantly with processing variables and throughout a single specimen: Fig. 2 shows low pore volume fraction regions with relatively smooth fracture surfaces, through to regions where the solid phase appears particulate resulting in a very rough fracture surface. The level 1 pore structure is also obvious in Fig. 1(b), which is a TEM micrograph presented at the same magnification as Fig. 1(a). The above micrographs suggest that the level 1 pores are the largest interconnected pores. Thus the characteristics of the level 1 pore system will strongly influence the mass transport properties of the geopolymer.

A series of bright-field TEM micrographs of sectioned grains of geopolymer 2.5/1.26 were recorded [see Figs. 1(b), 3 
and 4, and Maitland (2011)]. These show regions greater than several $\mu \mathrm{m}^{2}$ in size free from sectioning artefacts, which suggests that ultramicrotomy of resin-embedded grains is a suitable method for preparing geopolymer specimens - at least for geopolymer 2.5/1.26. Bell (2007) also reported success with this method. The attractions of this method are large $\left(>10 \mu \mathrm{m}^{2}\right)$ electron-transparent regions, uniform section thickness and the fact that many sections may be produced in a relatively short time compared to ion beam thinning methods. Ultramicrotoming of resin-embedded grains is not ideal though: artefacts such as torn sections and compression frac-
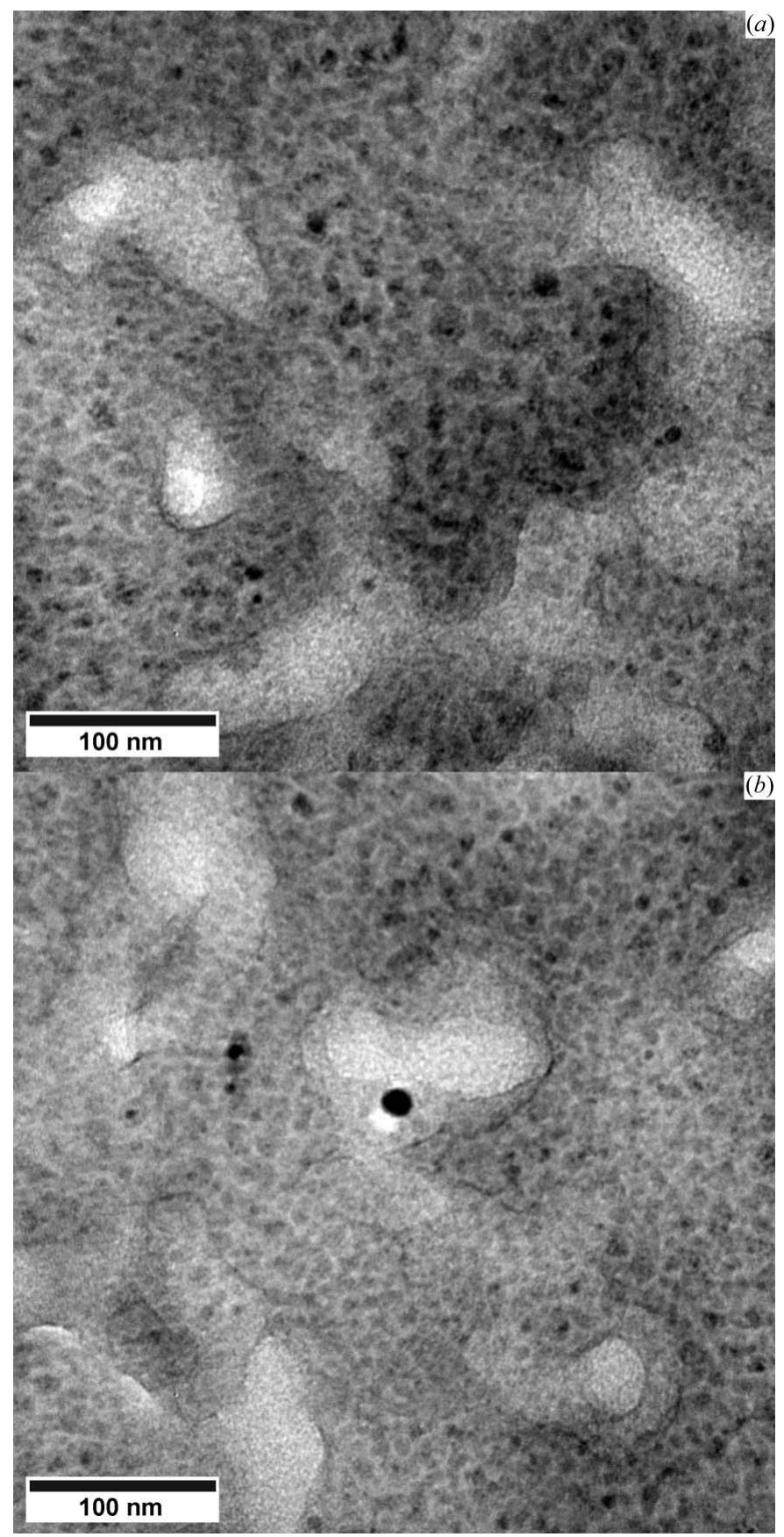

Figure 3

TEM micrographs of geopolymer 2.5/1.26 that exhibit both the level 1 and the level 2 pore structures. tures were observed; most grains embedded were less than $10 \mu \mathrm{m}$ in size and it is not known if larger grains will section successfully; and contributions to the image contrast from the resin in the micrographs can also be observed. It is unlikely that some regions of the section in Fig. 1(b) would have remained intact without support from the resin. Hence resin embedding is probably required to obtain unbiased observations of the pore structure with TEM.

In Figs. 3 and 4 there is non-uniform electron contrast in the region that complements the level 1 pore system. This suggests that a system of smaller pores exists, which is designated here
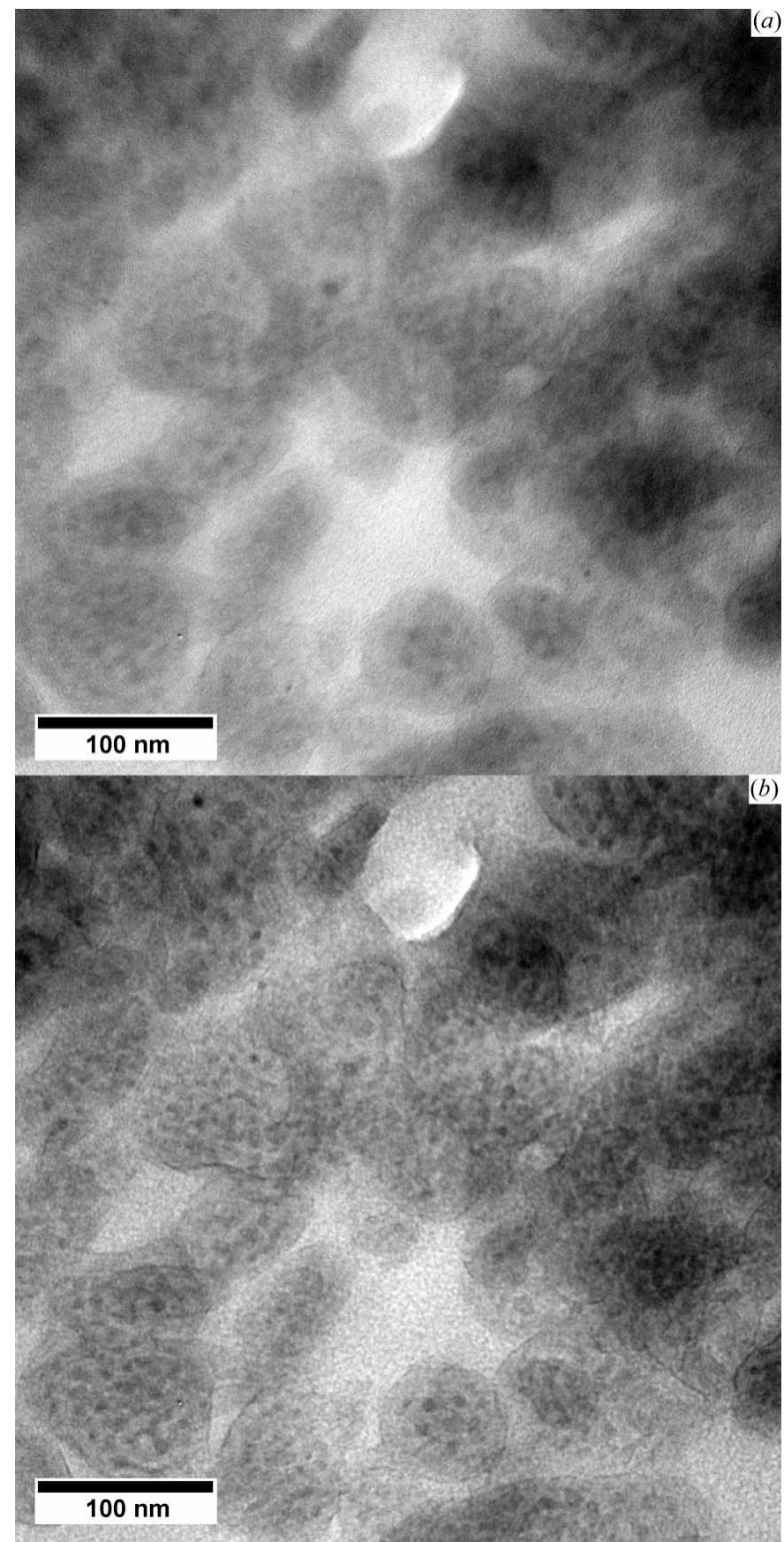

\section{Figure 4}

TEM micrographs of the same region of sample 2.5/1.26 recorded with different defocus: (a) $\Delta z \simeq 0 \mu \mathrm{m}$ and (b) $\Delta z \simeq-0.8 \mu \mathrm{m}$. 
as the level 2 pore system. This system was observed throughout the specimen. SANS experiments discussed later support the assumption that the non-uniform electron contrast is due to pores. Pores of this size range (pore diameter < $10 \mathrm{~nm}$ ) and occupying a significant volume fraction have been found in other metakaolin geopolymers by TEM (Kriven et al., 2004; Bell \& Kriven, 2004; Duxson et al., 2005). The structure has been described as clusters of particulates of aluminosilicate approximately $5-10 \mathrm{~nm}$ in diameter.

The micrographs in Fig. 4 are two of a defocus series recorded. It was concluded from examination of Fresnel fringes around the edges of the level 1 pores that Fig. 4(a) was in focus. Well defined level 2 pores or solid phase cannot be observed in the focused image. The carbon-supporting film and resin may also contribute to this lack of definition. Defocusing yields more defined regions of contrast (increased granularity) but this extra definition could be attributed to instrumental effects due to changes in the contrast transfer function. Determining information about the level 2 pore structure is complicated by these effects.

The micrographs in Fig. 3 were recorded from different regions of the geopolymer $2.5 / 1.26$ with $\Delta z$ of the same order as the defocused image in Fig. 4(b). The level 2 structure in Figs. 3 and $4(b)$ has a similar appearance, but the level 1 structure has a distinctly different morphology: the complement of the level 1 pores in Fig. 3 is more nearly continuous and does not have a particulate appearance as in Fig. 4. This variation in level 1 structure agrees with observations from the SEM micrographs above.

\subsection{Ultra-small-angle scattering: statistically significant morphological information}

The previous section presents some of the challenges involved in measuring morphological quantities that characterize the pore systems in geopolymers with electron microscopy. Electron microscopy cannot be disregarded though, as it is currently the only available technique for measuring higher-order descriptors on smaller length scales. Gaining a representative description of such a complex material, with possible modification of structure due to sample preparation and vacuum, poses real problems.

An investigation using USANS of a geopolymer-type material (referred to as 'alkali-activated cement') was reported by Phair et al. (2003). One geopolymer was made from alkali activator (potassium hydroxide and sodium silicate) mixed with fly ash and kaolin. USANS data were collected from specimens between 0.5 and $11.2 \mathrm{~mm}$ thick. Severe multiple scattering affected their data, making it challenging to determine structural information. This was limited to an abstract single scattering particle radius of approximately $4 \mu \mathrm{m}$. From the power law behaviour of their USANS curves it was concluded that the geopolymer structure exhibited fractal characteristics on the length scales probed by the USANS instrument $(\sim 20-0.1 \mu \mathrm{m})$.

Fig. 5 depicts the USANS curves from geopolymers 2.0/1.0, 2.5/1.2 and 2.8/1.4. No power law behaviour is exhibited over more than an order of magnitude in $q$. There is minimal difference between the scattering from all three samples for $0.005 \leq q \leq 0.1 \mathrm{~nm}^{-1}$, indicating that structure less than approximately $2 \pi / 0.005 \mathrm{~nm}^{-1}=1 \mu \mathrm{m}$ and greater than $\sim 60 \mathrm{~nm}$ in 'size' is similar on average. Comparing this size range with Figs. 1-4, and knowing that the shoulder is most likely due to a porous matrix, it seems that the morphology of the level 1 structure is not severely affected by changes in chemical composition over the range examined. The scattering curves in Fig. 5 are not in agreement for $q<0.004 \mathrm{~nm}^{-1}$; this suggests that the micrometre-sized structure varies more between the three geopolymers than the level 1 pore structure. Obtaining this statistically significant information from electron microscopy alone would be very difficult as a sufficient volume cannot be sampled easily, and the effects of dehydration and sample preparation would be unknown.

Only a basic analysis of the USANS data is given here. A more detailed analysis will need to consider the contribution from partially dissolved MK. The USANS curve of the MK precursor was recorded but it was severely affected by multiple scattering (sample cell was too thick) and was discarded. The observed variation in scattering over different $q$ ranges shows that USANS, when the specimens are sufficiently thin, is sensitive to changes in the structure of geopolymers over the range of compositions of interest.

\subsection{Dominant source of scattering}

It is important to determine the sources of the neutron scattering due to the heterogeneous, complex structure of geopolymers and the presence of impurities, e.g. scattering may be due to solid phases of different scattering length density, pores or a combination of both sources. It was

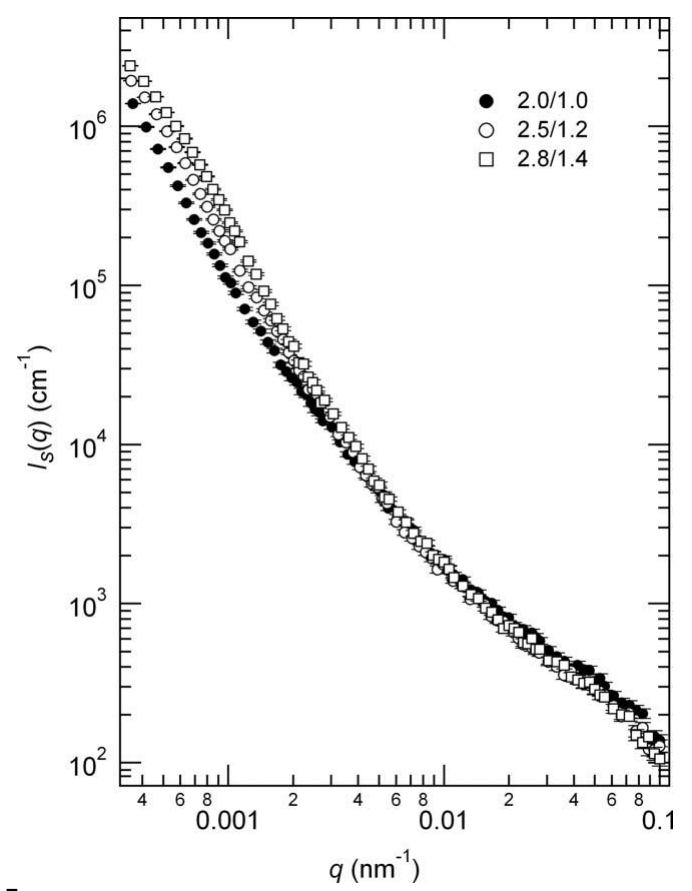

Figure 5

USANS of nondehydrated geopolymers. 
conjectured in a previous SANS investigation by Buckley et al. (2002) that the scattering was due to pores. To reduce multiple scattering, Phair et al. (2003) immersed their geopolymers in a $30 / 70(v / v)$ mixture of $\mathrm{D}_{2} \mathrm{O} / \mathrm{H}_{2} \mathrm{O}$, causing a reduction in the USANS by an order of magnitude.

SANS experiments were performed exclusively on geopolymer 2.5/1.2. The SANS curves of a specimen before and after dehydration are given in Fig. 6. If the complement of the level 1 pore system had a uniform neutron contrast, its surface was smooth, and the transition between solid and pore occurred in less than several tenths of a nanometre, a fit of the power law $I(q)=A q^{-\beta}$ for $q>q_{\mathrm{p}}$ would yield $\beta=4$. The rule (Ciccariello, 2003)

$$
q_{\mathrm{p}}=\frac{2 \pi}{\min (\bar{D}, \bar{d})}
$$

was used to estimate $q_{\mathrm{p}}$, where $\bar{D}$ and $\bar{d}$ are the mean diameter of the scattering particle and the distance between particles, respectively. The complement of the level 1 pore structure is nonparticulate so there is no particle diameter to directly apply [equation (2)]. To overcome this, it was assumed that equation (2) still holds if $\bar{D}$ and $\bar{d}$ are replaced by $\bar{l}_{1}$ and $\bar{l}_{\mathrm{c} 1}$, which are the mean chord of the level 1 pore system and the mean chord of the complement (if it had uniform neutron contrast) of the level 1 pore system, respectively. From visual inspection of Figs. 1, 3 and 4 it was estimated that $\min \left(\bar{l}_{1}, \bar{l}_{\mathrm{c} 1}\right)>20 \mathrm{~nm}$, thus $q_{\mathrm{p}}<0.3 \mathrm{~nm}^{-1}$. No fits of the power law for $q>0.3 \mathrm{~nm}^{-1}$ returned a value for $\beta$ close to 4 . A likely cause of $\beta \neq 4$, also taking into account the non-uniform electron contrast observed in the transmission electron

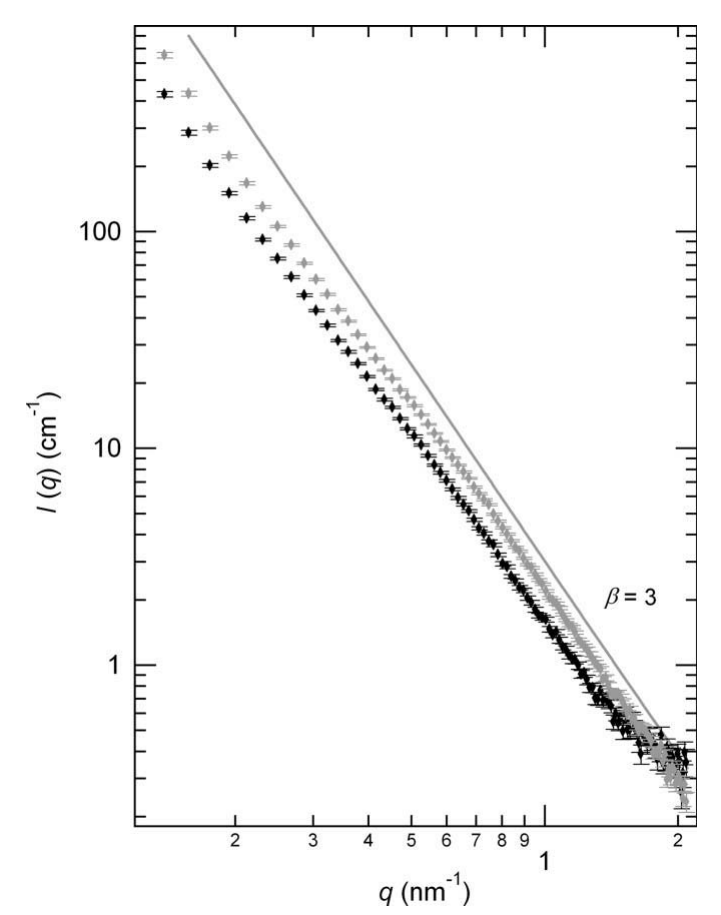

Figure 6

SANS from a specimen of geopolymer 2.5/1.2 before (black symbols) and after (grey symbols) dehydration in air at $423 \mathrm{~K}$. The solid grey line is a guide showing $I \propto q^{-3}$ power law scattering. micrographs (Figs. 3 and 4), is that within the complement of the level 1 pore system the neutron contrast is non-uniform. Considering the significant volume fraction of pores with widths less than $10 \mathrm{~nm}$ reported in other metakaolin geopolymers (Kriven, 2004; Kriven \& Bell, 2004; Duxson et al., 2005), it is likely that this non-uniform neutron contrast is due to pores. The observed SANS contains some contributions from the internal structure of the remnants of MK grains and this may also be affecting the value of $\beta$. If there is significant scattering from these MK grains in the SANS $q$ range, it will increase the uncertainty of any information derived from the shape of the SANS curve about the solid reaction product.

A series of contrast variation measurements were performed on geopolymer $2.5 / 1.2$ to confirm the source of the neutron scattering. The immersion of specimens of nondehydrated geopolymer 2.5/1.2 in different solvents caused the scattering to vary in magnitude by up to a factor of 80 (see Fig. 7). Similarly, immersion of dehydrated specimens of geopolymer 2.5/1.2 in different solvents caused the scattering to vary by up to a factor of 20 (see Fig. 8). Thus the scattering from both dehydrated and nondehydrated geopolymer 2.5/1.2 is dominated by that from a pore-solid system, and these pores are accessible to water. The change in shape of the scattering curves for $1.0<q<2.0 \mathrm{~nm}^{-1}$ after immersion is attributed to incoherent scattering from varying amounts of hydrogen in the solvent. As expected, the intensity of dehydrated 2.5/1.2 in air and immersed in a solvent with the same scattering length density as air are equal within the uncertainty.

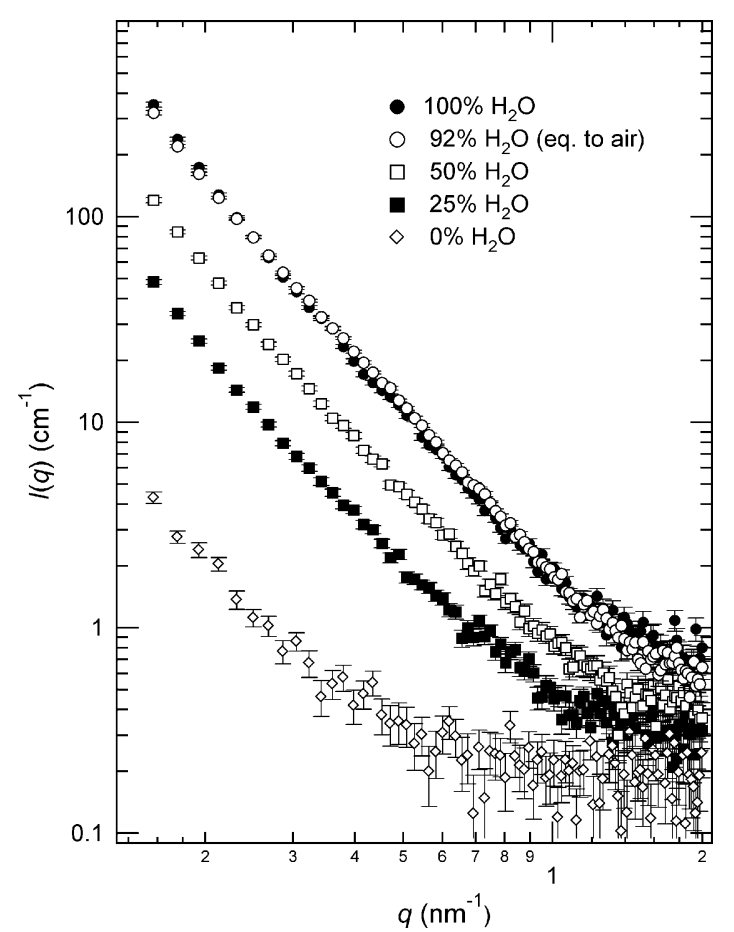

Figure 7

SANS from nondehydrated specimens of 2.5/1.2 immersed in solvents of different scattering length density. The legend gives the vol.\% of $\mathrm{H}_{2} \mathrm{O}$ in the solvents. 


\subsection{Scattering length density, residual water and the effects of dehydration}

An aim of this study was to measure the scattering length density of the solid phase of both dehydrated, $\rho$, and nondehydrated, $\rho_{\mathrm{w}}$, geopolymer. This would allow model intensities to be compared with experiment on an absolute scale. A comparison on an absolute scale is important since geopolymers are complex systems with relatively featureless scattering curves.

The contrast variation plots given in Fig. 9 consist of $I(q)$ at $q=0.176 \mathrm{~nm}^{-1}$ (from the data sets displayed in Figs. 7 and 8), versus the scattering length density of the solvent $\rho_{\text {sol }}$. For twophase systems they are parabolic curves with the location of the vertex equalling the scattering length density of the solid phase. The first estimate of the scattering length density of the solid phase of dehydrated 2.5/1.2 was calculated using the following expression derived from Porod (1982):

$$
I\left(q, \rho_{\mathrm{sol}}\right)=C(q)\left(\rho-\rho_{\mathrm{sol}}\right)^{2} \quad\left(\mathrm{~cm}^{-1}\right) .
$$

The positive parameter $C(q)$ is dependent on the pore morphology and independent of $\rho_{\text {sol }}$. Equation (3) is valid when the system can be considered two phase (open pores and solid), all scattering is due to open pores and no residual water is present. Fitting of equation (3) to the data from the dehydrated specimens resulted in $\rho=4.108(14) \times 10^{10} \mathrm{~cm}^{-2}$ and $C\left(0.176 \mathrm{~nm}^{-1}\right)=1.86(2) \times 10^{-19} \mathrm{~cm}^{3}$. As shown in Fig. 9, the fitted values are likely to be inaccurate owing to the poor agreement of the model. The uncertainties of all the fitted coefficients were calculated by the nonlinear least-squares

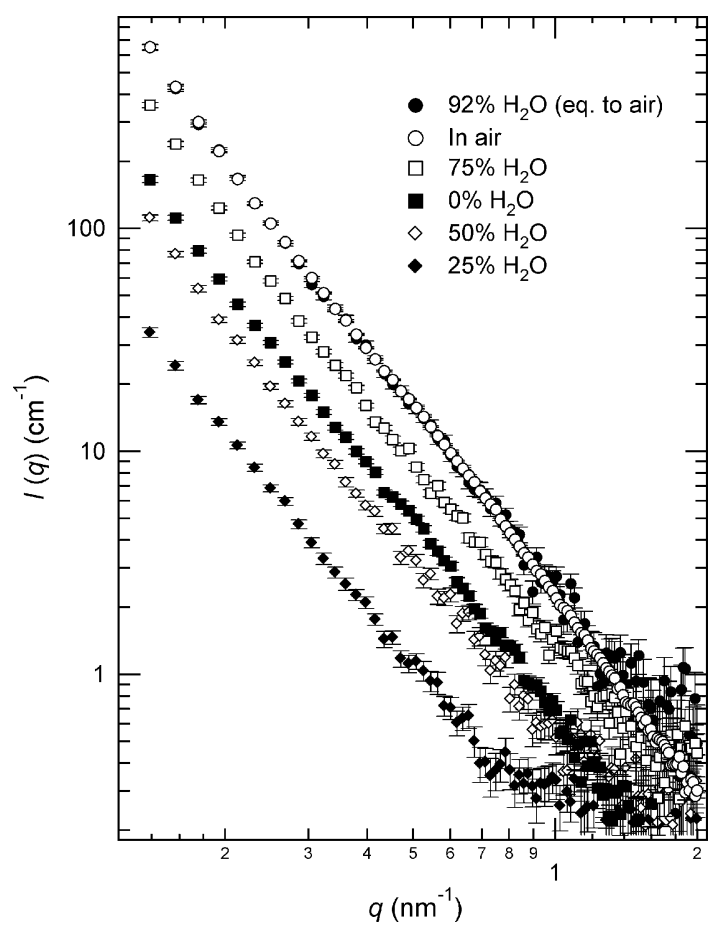

Figure 8

SANS from dehydrated specimens of geopolymer 2.5/1.2 immersed in solvents of different scattering length density and in air. The legend gives the vol. $\%$ of $\mathrm{H}_{2} \mathrm{O}$ in the solvents. routine of Igor Pro (http://www.wavemetrics.com). These may be underestimated as their calculation only included the uncertainty of intensity values in the contrast variation plots and did not include other sources such as the uncertainty of the solvent scattering length density. An estimate of the solid phase scattering length density of $3.4 \times 10^{10} \mathrm{~cm}^{-2}$ was calculated using the atomic density of 0.067 atoms $\AA^{-3}$ measured by X-ray scattering (Rowles, 2004) and composition $\mathrm{Na}_{n}\left[-\left(\mathrm{SiO}_{2}\right)_{3.2}-\left(\mathrm{AlO}_{2}\right)-\right]_{n} \quad$ (Rowles \& O'Connor, 2009) measured by SEM energy dispersive spectrometry. This composition was measured from the matrix region of an Si:Al:Na = 2.5:1:1.29 geopolymer. The scattering length density determined using equation (3) is significantly higher than the calculated value of $3.4 \times 10^{10} \mathrm{~cm}^{-2}$. There are $\mathrm{Ca}$, $\mathrm{Mg}, \mathrm{Ti}, \mathrm{Fe}$ and $\mathrm{Zr}$ impurities present in the geopolymers, but these will have little influence on $\rho$ as their individual concentration is below $1 \mathrm{wt} \%$.

The nonzero minimum in the contrast variation plot (Fig. 9) of the dehydrated geopolymer suggests the existence of a third phase. This third phase could be level 1 and/or level 2 pores that were inaccessible to the solvent during immersion. The contrast variation plot of the nondehydrated sample is not significantly greater than zero. Upon dehydration there may be a shrinkage of the solid phase that restricts the accessibility of the solvent to some pore space, and hence some closed (using the current immersion method) pores are formed. This pore space, originally containing water, would contain air after dehydration. Hence it is assumed that the scattering length density of these closed pores is zero.

Early contributions to three-phase theory were made by Peterlin (1965), Stuhrman \& Kirste (1965), Goodisman \& Brumberger (1971) and Wu (1982). From expressions given by Ciccariello \& Riello (2007) for general three-phase systems, the scattering from the model described above is

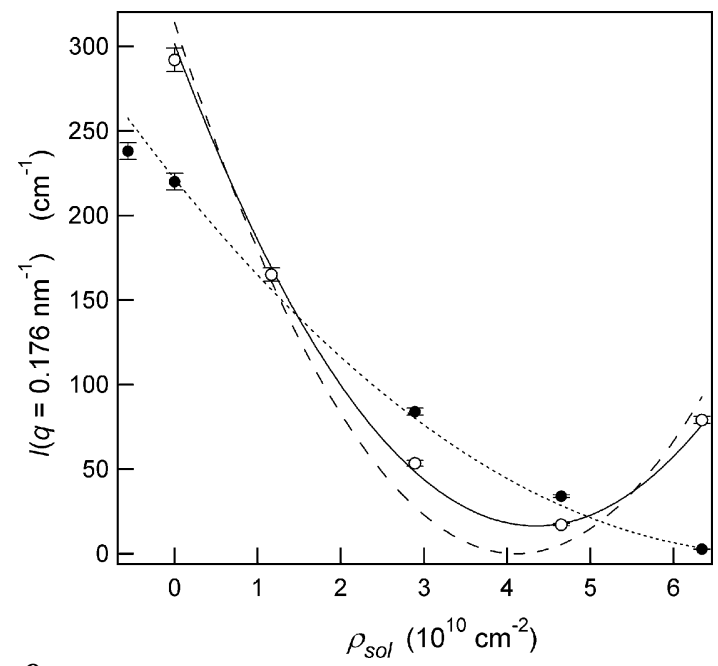

Figure 9

Contrast variation plots of nondehydrated (solid circles) and dehydrated (open circles) geopolymer 2.5/1.2. The fits of equations (3) and (4) are given by the dashed and solid lines, respectively. The fit of equation (9) is given by the dotted line. 


$$
\begin{aligned}
I\left(q, \rho_{\mathrm{sol}}\right)= & \rho^{2} \varphi_{\mathrm{s}}\left(1-\varphi_{\mathrm{s}}\right) \tilde{\Gamma}_{\mathrm{s}}(q)-\rho\left[\varphi_{\mathrm{s}}\left(1-\varphi_{\mathrm{s}}\right) \tilde{\Gamma}_{\mathrm{s}}(q)\right. \\
& \left.+\varphi_{\mathrm{o}}\left(1-\varphi_{\mathrm{o}}\right) \tilde{\Gamma}_{\mathrm{o}}(q)-\varphi_{\mathrm{c}}\left(1-\varphi_{\mathrm{c}}\right) \tilde{\Gamma}_{\mathrm{c}}(q)\right] \rho_{\text {sol }} \\
& +\varphi_{\mathrm{o}}\left(1-\varphi_{\mathrm{o}}\right) \tilde{\Gamma}_{\mathrm{o}}(q) \rho_{\mathrm{sol}}^{2} \\
= & \alpha(q)+\beta(q) \rho_{\mathrm{sol}}+\gamma(q) \rho_{\mathrm{sol}}^{2} \quad\left(\mathrm{~cm}^{-1}\right) .
\end{aligned}
$$

Here $\varphi_{i}$ and $\tilde{\Gamma}_{i}(q)$ are the volume fraction and Fourier transform of the isotropic autocorrelation function of the $i$ th phase, respectively. The subscripts s, o and c denote the solid phase, open pores and closed pores, respectively. These pores may not be open or closed to all water under all conditions, but are defined here by whether they fill with the solvent under the current experimental conditions. Equation (4) cannot be used directly without a priori information to determine $\rho$ from the contrast variation plot since it is only a quadratic function of $\rho_{\text {sol. }}$.

The coefficients $\alpha, \beta$ and $\gamma$ were determined by fitting a quadratic function to 48 contrast variation plots, one for each $q$ value. The scattering from the open pores was then obtained directly since $\gamma(q)=\varphi_{0}\left(1-\varphi_{0}\right) \tilde{\Gamma}_{o}(q)$ and is presented in Fig. 10. One can also directly examine the $q$ dependence of the scattering of the solid phase as $\alpha(q) \propto \varphi_{\mathrm{s}}\left(1-\varphi_{\mathrm{s}}\right) \tilde{\Gamma}_{\mathrm{s}}(q)$. To obtain the scattering from the closed and solid phases requires knowledge of $\rho$ :

$$
\varphi_{\mathrm{s}}\left(1-\varphi_{\mathrm{s}}\right) \tilde{\Gamma}_{\mathrm{s}}(q)=\alpha(q) / \rho^{2}
$$

and

$$
\varphi_{\mathrm{c}}\left(1-\varphi_{\mathrm{c}}\right) \tilde{\Gamma}_{\mathrm{c}}(q)=\alpha(q) / \rho^{2}+\beta(q) / \rho+\gamma(q)
$$

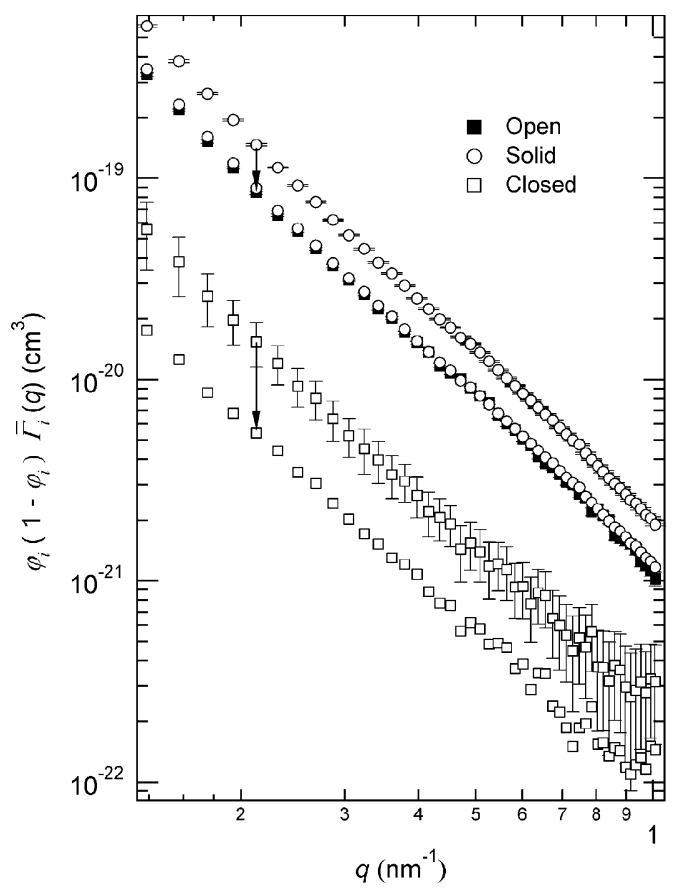

Figure 10

Scattering $\varphi_{i}\left(1-\varphi_{i}\right) \tilde{\Gamma}_{i}(q)$ from the solid phase and closed pores over the range of likely $\rho$ as calculated using the three-phase model: the tail of an arrow shows the scattering if $\rho=3.4 \times 10^{10} \mathrm{~cm}^{-2}$, the tip of an arrow that if $\rho=4.35 \times 10^{10} \mathrm{~cm}^{-2}$. Scattering from open pores $\varphi_{\mathrm{o}}\left(1-\varphi_{\mathrm{o}}\right) \tilde{\Gamma}_{\mathrm{o}}(q)$ is also given; it is determined independently of $\rho$. For clarity, uncertainties of the solid and closed intensities are only given on one curve.
Fig. 10 presents $\varphi_{\mathrm{s}}\left(1-\varphi_{\mathrm{s}}\right) \tilde{\Gamma}_{\mathrm{s}}(q)$ and $\varphi_{\mathrm{c}}\left(1-\varphi_{\mathrm{c}}\right) \tilde{\Gamma}_{\mathrm{c}}(q)$ calculated using the complementary value of $\rho=3.4 \times 10^{10} \mathrm{~cm}^{-2}$.

It is also possible to calculate an upper limit of $\rho=4.42(8) \times$ $10^{10} \mathrm{~cm}^{-2}$ using the current model. $\tilde{\Gamma}_{i}(q)$ are defined such that $\int_{0}^{\infty} q^{2} \tilde{\Gamma}_{i}(q) \mathrm{d} q=2 \pi^{2}$, and note that $\tilde{\Gamma}_{\mathrm{s}}(q)$ is independent of $\varphi_{\mathrm{c}}$. Consider the system (denoted by a prime) where there is no closed porosity $\varphi_{\mathrm{c}^{\prime}}=0$; it follows from Babinet's principle that $\tilde{\Gamma}_{\mathrm{o}^{\prime}}(q)=\tilde{\Gamma}_{\mathrm{s}}(q)$. The approximation was made that, for $\varphi_{\mathrm{c}} \ll 1$, $\tilde{\Gamma}_{\mathrm{o}}(q) \simeq \tilde{\Gamma}_{\mathrm{o}^{\prime}}(q)=\tilde{\Gamma}_{\mathrm{s}}(q)$. It is also reasonable to assume that $\varphi_{\mathrm{s}}>0.5$. With these assumptions and equation (4) one obtains the condition $\rho^{2}<\alpha(q) / \gamma(q) \simeq$ constant. For comparison, the scattering from the individual phases for $\rho$ close to this limit is also given in Fig. 10. The scattering from all three phases had essentially the same $q$ dependence within the uncertainties for a range of $\rho$. Owing to the large relative uncertainty of $\varphi_{\mathrm{c}}\left(1-\varphi_{\mathrm{c}}\right) \tilde{\Gamma}_{\mathrm{c}}(q)$, the comparison of its shape with the other scattering curves is rather approximate.

To determine the scattering length density of the solid phase in the nondehydrated geopolymer, it was approximated as a two-phase system. This approximation is justified by the minimum in the contrast variation plot (Fig. 9) being close to zero. Equation (3) was modified to account for residual pore water:

$$
I_{\mathrm{w}}(q)=C_{\mathrm{w}}(q)\left(\rho_{\mathrm{w}}-\rho_{\text {mix }}\right)^{2} \quad\left(\mathrm{~cm}^{-1}\right) .
$$

In this case, after adding the solvent the pores are filled with a mixture of solvent and residual pore solution. The parameters describing the nondehydrated specimen are denoted with a subscript ' $w$ '. It is assumed below that the residual pore solution is $\mathrm{H}_{2} \mathrm{O}$. In reality the pore solution contains $\mathrm{Na}^{+}$ions and possibly other molecules. The mixture of solvent and residual water will have a scattering length density equal to

$$
\rho_{\text {mix }}=\left(1-\varphi_{\text {sol }}\right) \rho_{\mathrm{H}_{2} \mathrm{O}}+\varphi_{\text {sol }} \rho_{\text {sol }} \quad\left(\mathrm{cm}^{-2}\right),
$$

where $\varphi_{\text {sol }}$ is the volume fraction of the mixture occupied by the solvent and $\rho_{\mathrm{H}_{2} \mathrm{O}}$ is the scattering length density of liquid $\mathrm{H}_{2} \mathrm{O}$. The volume fraction of the pore space in the nondehydrated geopolymer occupied by residual water prior to the geopolymer being immersed in the solvent is defined as $\varphi_{\text {res }}$. If the solvent only filled the empty pore space within the geopolymer then $\varphi_{\text {res }}=1-\varphi_{\text {sol }}$. The empty space in the SANS sample cell (not occupied by the disc of geopolymer) was also filled with solvent during the experiment. Hence $\varphi_{\text {sol }}$ will be greater than or equal to the volume fraction of the pore space in the geopolymer filled with solvent. Thus, only a lower limit on $\varphi_{\mathrm{res}}$ is provided by $\varphi_{\mathrm{sol}}: \varphi_{\mathrm{res}} \geq 1-\varphi_{\mathrm{sol}}$. Substituting $\rho_{\mathrm{H}_{2} \mathrm{O}}=-0.56 \times 10^{10} \mathrm{~cm}^{-2}$ and equation (8) into (7) allows for determination of $\varphi_{\text {sol }}$ and the scattering length density $\rho_{\mathrm{w}}$ :

$$
\begin{aligned}
I_{\mathrm{w}}\left(q, \rho_{\mathrm{sol}}\right)= & C_{\mathrm{w}}(q)\left[\rho_{\mathrm{w}}+0.56 \times 10^{10}\right. \\
& \left.-\varphi_{\text {sol }}\left(\rho_{\text {sol }}+0.56 \times 10^{10}\right)\right]^{2} \quad\left(\mathrm{~cm}^{-1}\right) .
\end{aligned}
$$

It is not possible to independently fit equation (9) to the contrast variation plot of the nondehydrated specimens as the correlation between fitted parameters is \pm 1 . The mass of nondehydrated specimens of geopolymer 2.5/1.2 was measured after drying in the air of the SANS instrument 
room, after saturation with solvents of different scattering length density and then after drying in air at $423 \mathrm{~K}$. From these mass measurements, and using a method similar to an Archimedes-type determination of open porosity, it was calculated that $\varphi_{\text {res }} \leq 0.388(8)$. [See Maitland (2011) for details of this calculation.] This gravimetric based limit on $\varphi_{\text {res }}$ implies that $\varphi_{\text {sol }} \geq 1-0.388(8)=0.612(8)$. Using equation (9), this corresponds to $\rho_{\mathrm{w}} \geq 4.21(6) \times 10^{10} \mathrm{~cm}^{-2}$; this value is much higher than the expected value of approximately $3.4 \times 10^{10} \mathrm{~cm}^{-2}$. This disagreement may be due to chemical species in the geopolymer that are mixing or exchanging with the solvent.

The plots in Fig. 6 show that dehydration has a significant effect on the SANS: there is minimal change in the shape of the scattering curve (as observed from the $\log I$ versus $\log q$ plots) upon dehydration, but a scaling along the vertical and/ or horizontal axes seems to have occurred. The subtle differences in the shape for $q>1.2 \mathrm{~nm}^{-1}$ are due to less water being present in the $423 \mathrm{~K}$ specimen, causing a reduction in incoherent scattering from hydrogen. The minimal change in the shape of the scattering shows that no severe change in the morphology on the length scale probed by the SANS data has occurred. Analysis of the SANS data to elucidate whether the change in scattering was due to shrinkage and/or a change in scattering length density of the solid phase is in progress.

\section{Conclusion}

It has been demonstrated that ultramicrotoming of resinembedded grains produces uniform thin sections of geopolymer suitable for TEM. Geopolymer 2.5/1.26 contained a bilevel pore structure as seen in SEM and TEM micrographs. TEM and SEM clearly showed that the morphology of the larger level 1 pore system varied significantly throughout this geopolymer. The appearance of the smaller level 2 pore system in TEM micrographs was strongly dependent on focus conditions. The shape of the USANS and SANS curves supported the existence of the bi-level pore structure. USANS data showed that geopolymers 2.0/1.0, 2.5/1.2 and 2.8/1.4 had statistically different micrometre-sized structures, but very similar structure on the $1 \mu \mathrm{m}-60 \mathrm{~nm}$ length scale. It was confirmed from contrast variation experiments that the source of SANS from geopolymer 2.5/1.2 was a pore system. Contrast variation plots suggested that some of the pores in the dehydrated 2.5/1.2 were closed under the current experimental conditions. A three-phase analysis was developed, and the scattering from the solid, open pore and closed pore phases was determined as a function of the scattering length density of the solid phase $\rho$. The scattering from all three phases had the same $q$ dependence within the uncertainties over the range of likely $\rho$. This analysis also provided an upper limit for $\rho$ of $4.42(8) \times 10^{10} \mathrm{~cm}^{-2}$. The determination of the scattering length density of the nondehydrated geopolymer $\rho_{\mathrm{w}}$ was hampered by the presence of residual water. A lower limit of $4.21(6) \times 10^{10} \mathrm{~cm}^{-2}$ for $\rho_{\mathrm{w}}$ was calculated using a gravimetrically determined value for $\varphi_{\text {res }}$. The expected value of the scattering length density of the solid phase is approximately
$3.4 \times 10^{10} \mathrm{~cm}^{-2}$ and is significantly lower than this limit. It is possible that other chemical species are mixing and or exchanging with the solvent. SANS could provide a method to probe these mobile species. The contrast variation data also suggested that the closed pore volume fraction is relatively small. Owing to the limited $q$ range of the current data it was not possible to quantify the open and closed pore volume fractions. SANS from dehydrated and nondehydrated geopolymer 2.5/1.2 showed that dehydration does not cause a severe change in morphology of the nanoporosity on the length scale probed. A change in scattering was observed and this change could be explained by a uniform shrinkage of the structure or a change in scattering length density of the solid phase or both. Analysis of this data to determine which of these has occurred is in progress. The findings above provide useful information for the construction and validation of structural models of metakaolin-based geopolymers.

CFM thanks the Australian government for an Australian Postgraduate Award, the Australian Institute for Nuclear Science and Engineering for a Postgraduate Research Award, and the Centre for Sustainable Resource Processing for a Postgraduate Research Scholarship. The authors acknowledge the facilities and the scientific and technical assistance of the Australian Microscopy \& Microanalysis Research Facility at the Centre for Microscopy, Characterization and Analysis (CMCA), The University of Western Australia, a facility funded by the university, state and Commonwealth governments. We thank John Murphy of CMCA for ultramicrotoming of the geopolymer specimens. We acknowledge Joan Connolly whilst at Curtin University for initiating the USANS investigation and Matthew Rowles for production of several geopolymers. Elliot Gilbert and Jamie Schulz of the Australian Nuclear Science and Technology Organization are thanked for their assistance with the SANS experiments. We thank Salvino Ciccariello and an anonymous referee for suggestions regarding the three-phase analysis.

\section{References}

Barker, J. G., Glinka, C. J., Moyer, J. J., Kim, M. H., Drews, A. R. \& Agamalian, M. (2005). J. Appl. Cryst. 38, 1004-1011.

Bell, J. L. (2007). Personal communication.

Bell, J. L., Gordon, M. \& Kriven, W. M. (2006). Microsc. Microanal. 12(Suppl. 2), 552CD-553CD.

Bell, J. L. \& Kriven, W. M. (2004). Microsc. Microanal. 10, 590-591.

Buckley, C. E., O' Connor, B. \& Rowles, M. (2002). AINSE Progress

Report for 02/014. Australian Institute of Nuclear Science and Engineering, Lucas Heights, NSW, Australia.

Ciccariello, S. (2003). Fibres Text. Eastern Eur. 11, 54-60.

Ciccariello, S. \& Riello, P. (2007). J. Appl. Cryst. 40, 282-289.

Davidovits, J. (1991). J. Therm. Anal. 37, 1633-1656.

Duxson, P., Fernandez-Jimenez, A., Provis, J. L., Lukey, G. C., Palomo, A. \& Van Deventer, J. S. J. (2007). J. Mater. Sci. 42, $2917-$ 2933.

Duxson, P., Mallicoat, S. L., Lukey, G. C., Kriven, W. M. \& van Deventer, J. S. J. (2007). Colloids Surf. A, 292, 8-20.

Duxson, P., Provis, J. L., Lukey, G. C., Mallicoat, S. L., Kriven, W. M.

\& Van Deventer, J. S. J. (2005). Colloids Surf. A, 269, 47-58.

Goodisman, J. \& Brumberger, H. (1971). J. Appl. Cryst. 4, 347-351. 
Gourley, J. T. \& Johnson, G. B. (2005). GGC2005 International Workshop on Geopolymers and Geopolymer Concrete, 2829 September, Curtin University, Perth, Western Australia, Australia.

Hardjito, D., Wallah, S. E., Sumajouw, D. M. J. \& Rangan, B. V. (2005). Aust. J. Struct. Eng. 6, 77-85.

Hos, J. P., McCormick, P. G. \& Byrne, L. T. (2002). J. Mater. Sci. 37, 2311-2316.

Kline, S. R. (2006). J. Appl. Cryst. 39, 895-900.

Kriven, W. M. (2004). Geopolymers: Recent Work and Future Directions, http://www.porotech.net/images/ACERS2004.pdf.

Kriven, W. M. \& Bell, J. L. (2004). 28th International Conference on Advanced Ceramics and Composites B, Ceramic Engineering and Science Proceedings, Vol. 25, pp. 99-104. Westerville: American Ceramic Society.

Kriven, W. M., Bell, J. L. \& Gordon, M. (2003). Advances in Ceramic Matrix Composites IX, Ceramic Transactions, Vol. 153, pp. 227-250. Westerville: American Ceramic Society.

Kriven, W. M., Gordon, M. \& Bell, J. L. (2004). Microsc. Microanal. 10(Suppl 2), 404-405.

Lukey, G. C. \& Van Deventer, J. S. J. (2005). GGC2005 International Workshop on Geopolymers and Geopolymer Concrete, 2829 September, Curtin University, Perth, Western Australia, Australia.

Maitland, C. F. (2011). PhD thesis, Curtin University of Technology, Perth, Western Australia, Australia. In preparation.

Palomo, A., Grutzeck, M. W. \& Blanco, M. T. (1999). Cem. Concr. Res. 29, 1323-1329.
Perera, D. S., Uchida, O., Vance, E. R. \& Finnie, K. S. (2007). J. Mater. Sci. 42, 3099-3106.

Perera, D. S., Vance, E. R., Aly, Z., Finnie, K. S., Hanna, J. V., Nicholson, C. L., Trautman, R. L. \& Stewart, M. W. A. (2003). ASME Conf. Proc. 2003, 1807-1814.

Peterlin, A. (1965). Makromol. Chem. 87, 152-155.

Phair, J. W., Schulz, J. C., Bertram, W. K. \& Aldridge, L. P. (2003). Cem. Concr. Res. 33, 1811-1824.

Porod, G. (1982). Small-Angle X-ray Scattering, edited by O. Glatter \& O. Kratky, pp. 17-51. New York: Academic Press.

Rahier, H., van Mele, B. \& Wastiels, J. (1995). J. Mater. Sci. 31, 8085.

Rowles, M. (2004). PhD thesis, Curtin University of Technology, Perth, Western Australia, Australia.

Rowles, M. R., Hanna, J. V., Pike, K. J., Smith, M. E. \& O' Connor, B. H. (2007). Appl. Magn. Reson. 32, 663-689.

Rowles, M. R. \& O' Connor, B. H. (2003). J. Mater. Chem. 13, 11611165.

Rowles, M. R. \& O'Connor, B. H. (2009). J. Am. Ceram. Soc. 92, 2354-2361.

Spurr, A. R. (1969). J. Ultrastruct. Res. 26, 31-43.

Stuhrman, H. B. \& Kirste, R. G. (1965). Z. Phys. Chem. Frankfurt, 46, $247-250$.

Subaer (2004). PhD thesis, Curtin University, Perth, Western Australia, Australia.

Vance, E. R., Hadley, J. H., Hsu, F. H. \& Drabarek, E. (2007). J. Am. Ceram. Soc. 91, 664-666.

Wu, W. (1982). Polymer, 23, 1907-1912. 